\title{
Evolved Gas Analysis-Mass Spectrometry (EGA-MS) Using Skimmer Interface System Equipped with Pressure Control Function
}

\author{
Tadashi ARI**
}

(Received January 4, 2005; Accepted April 10, 2005)

\begin{abstract}
A new evolved gas analysis-mass spectrometry (EGA-MS) equipped with a skimmer interface system and a pressure control function (PCF) has been developed successfully. The skimmer interface system based upon jet separator principle is constructed between a sample chamber surrounded by an electrical furnace and a vacuum chamber. Although the skimmer type is principally more excellent than a capillary type, it is a significant problem that the sensitivity of EGA-MS falls with increasing the temperature. The fall of detection sensitivity of EGA-MS depending on temperature rising has been remarkably improved by introducing the pressure control function (PCF). As the result of evaluating PCF by using the thermal decomposition of calcium oxalate monohydrate, the ionic intensities of the evolved gases increased more than twice. The new EGA-MS was applied to the thermal decomposition of zinc acetylacetonate monohydrate and polymethylmethacrylate (PMMA). It was confirmed that the more sensitive and precise gas analysis which prevented condensation of evolution gases and/or generation of by-products can be performed, by comparing with the capillary interface system of TG-MS.
\end{abstract}

\section{Introduction}

The study of compound decomposition by thermal analysis can be greatly enhanced when evolved gas analysis (EGA) used. EGA is known as one of thermal analysis methods for measuring the amount of generated gases from a sample as a function of temperature when the temperature of the sample is increased at a predetermined heating rate. For instance, this can be achieved by combination of thermogravimetry (TG) with other techniques capable of providing both qualitative and quantitative information. ${ }^{1-4)}$ It appears that most popular EGA technique is in practical use with mass spectrometry (MS) on account of its sensitivity, versatility and fast analysis time.

In EGA system, thermal analysis instrument provides, among other things, with precise and reproducible heating conditions. The gaseous products generated by volatilization, sublimation or chemical reaction, are flushed out of the furnace chamber with the help of a purge gas. The evolved gas is introduced in MS detector through a coupling system, which acts both as MS inlet and a pressure-reduction system. The coupling system should meet several specifications such as: rapid evolved-gas transfer from sample pan in furnace to MS; no memory effects; no degradation and secondary reaction or condensation of evolved gases; no interferences on the specifications of MS; no air insertion to MS during furnace opening; simplicity in construction; easy system decoupling and cleaning; low cost; and continuous monitoring and versatility with respect to MS. In addition, high sensitivity and repeatable flow conditions are necessary. In the con-

* Strategic Business Unit Thermal Analysis Group, Rigaku Corporation (3-9-12 Matsubara, Akishima, Tokyo 1968666, Japan)

Tel: 81-42-454-8126, Fax: 81-42-544-9650

E-mail: t-arii@rigaku.co.jp ventional skimmer interface system, principally, the pressure in the MS chamber and between the two concentric tubes change toward high vacuum pressures with increasing the temperature, so that the detection sensitivity of the MS is changed and is reduced notably in a wide temperature range. The reduction of detection sensitivity caused by the temperature variation of the gaseous species introduced into the MS chamber has not been hitherto treated in detail. However, it can be considered that this is one of significant problems of the previous EGA-MS equipments with the skimmer interface system.

A great variety of EGA-MS systems has been presented in literatures during the past decade and reviews ${ }^{5), 6)}$ have been written on the advantages, disadvantages, and applications. The coupling of a predominantly ambient pressure technique (TG, for example) to MS operated under high vacuum requires the circumvention of many problems or the development of unique approaches which may introduce new disadvantages. Thus, the effort to obtain chemical information from thermal processes using MS has resulted in a variety of instrumental designs and approaches.

Generally, EGA-MS type is roughly classified into two kinds of gas interface systems from the difference in interface structure which connects between a sample chamber and a vacuum chamber: capillary and skimmer interface systems. ${ }^{7), 8)}$ Nowadays, many EGA-MS systems have adapted the capillary interface system because the instrumental design becomes generally simple. It can succeed by modifications of commercial thermal analysis apparatus without interferences on the specifications, so that EGA-MS using capillary interface system so as represented with typical TG-MS equipment is commercially available.9)-11) On the other hand, although skimmer interface system offers principally significant advantages over capillary interface system, the instrumental design is complicated and fundamental studies are particularly scarce in 
practical use.

The purpose of this work is to propose a new type of EGA-MS equipment which adapted a pressure control function (PCF) devised in order to improve the serious disadvantage of skimmer interface system. The results of performance evaluation through applying of some different field samples can be a basis for design improvements. Furthermore, the ability of the EGA-MS was confirmed by comparing with the results of capillary interface system used by TG-MS.

\section{Experimental}

\subsection{Materials}

The powder samples of calcium oxalate monohydrate $\left(\mathrm{CaC}_{2} \mathrm{O}_{4} \cdot \mathrm{H}_{2} \mathrm{O}\right.$, purity $<98.5 \%$, Kanto Chemical Co., Inc.) and zinc acetylacetonate monohydrate $\left(\mathrm{C}_{10} \mathrm{H}_{14} \mathrm{O}_{4} \mathrm{Zn} \cdot \mathrm{H}_{2} \mathrm{O}\right.$, purity $<98.0 \%$, Wako Pure Chemical Industries, Ltd.) used were the commercially available reagent without any further purification. The pellet sample of polymethylmethacrylate (PMMA) was supplied by Kanto Chemical Co., Inc. as Cica-Reagent material. The $\mathrm{X}$-ray diffraction spectra showed only crystalline $\mathrm{CaC}_{2} \mathrm{O}_{4} \cdot \mathrm{H}_{2} \mathrm{O} .{ }^{12)}$ and $\mathrm{C}_{10} \mathrm{H}_{14} \mathrm{O}_{4} \mathrm{Zn} \cdot \mathrm{H}_{2} \mathrm{O} .{ }^{13)}$

The samples were weighed into a platinum crucible, and were heated in high-purity dry helium gas (99.999\%), at a flow rate of $300 \mathrm{~mL} / \mathrm{min}$.

\subsection{Apparatus}

2.2.1 EGA-MS The EGA-MS equipment (Model TPD type R, Rigaku) is comprised of three major components: quadrupole mass spectrometer, infrared image furnace, and gas sampling system. The EGA-MS with the unique gas sampling system called skimmer interface structure is shown in Fig. 1. It consists of two concentric quartz tubes with orifices which connected the two devices at atmospheric and vacuum pressures, for continuous and immediate sampling of the gases evolved from the samples. An intermediate pressurereduced chamber is provided between the sample and the MS chambers at atmospheric and vacuum pressures, respectively. A first orifice is formed between the intermediate pressure-reduced chamber and the sample chamber, and a second orifice is formed between the intermediate pressure-reduced chamber and the MS chamber. The gas generated in the sample chamber is controlled through these two orifices, and is introduced into the MS chamber.

Since the carrier gas used was also high-purity helium, the evolved gaseous species with higher masses were enriched after the second orifice, in consequence of the principle of a jet separator, i.e., the second orifice ensures that the composition of the molecular beam is identical to that of the gas presented in the sample chamber. Typically, with raising the temperature up to maximum $1200^{\circ} \mathrm{C}$ the pressure between the two quartz tubes was varied at 100-1,000 Pa using a rotary pump; the pressure at the ion source was varied at $5.0 \sim 40 \times 10^{-4} \mathrm{~Pa}$ with a turbo molecular pump. These two orifices in the quartz tubes and the specimen are covered with a platinum pipe which adsorbs infrared beam from the furnace in order to allow the uniform heating and to avoid the condensation of the evolved gas in the orifices. The maximum use temperature of the interface is in excess of $1,200^{\circ} \mathrm{C}$. The pressure value of the ion gauge (I.G) in the MS chamber is

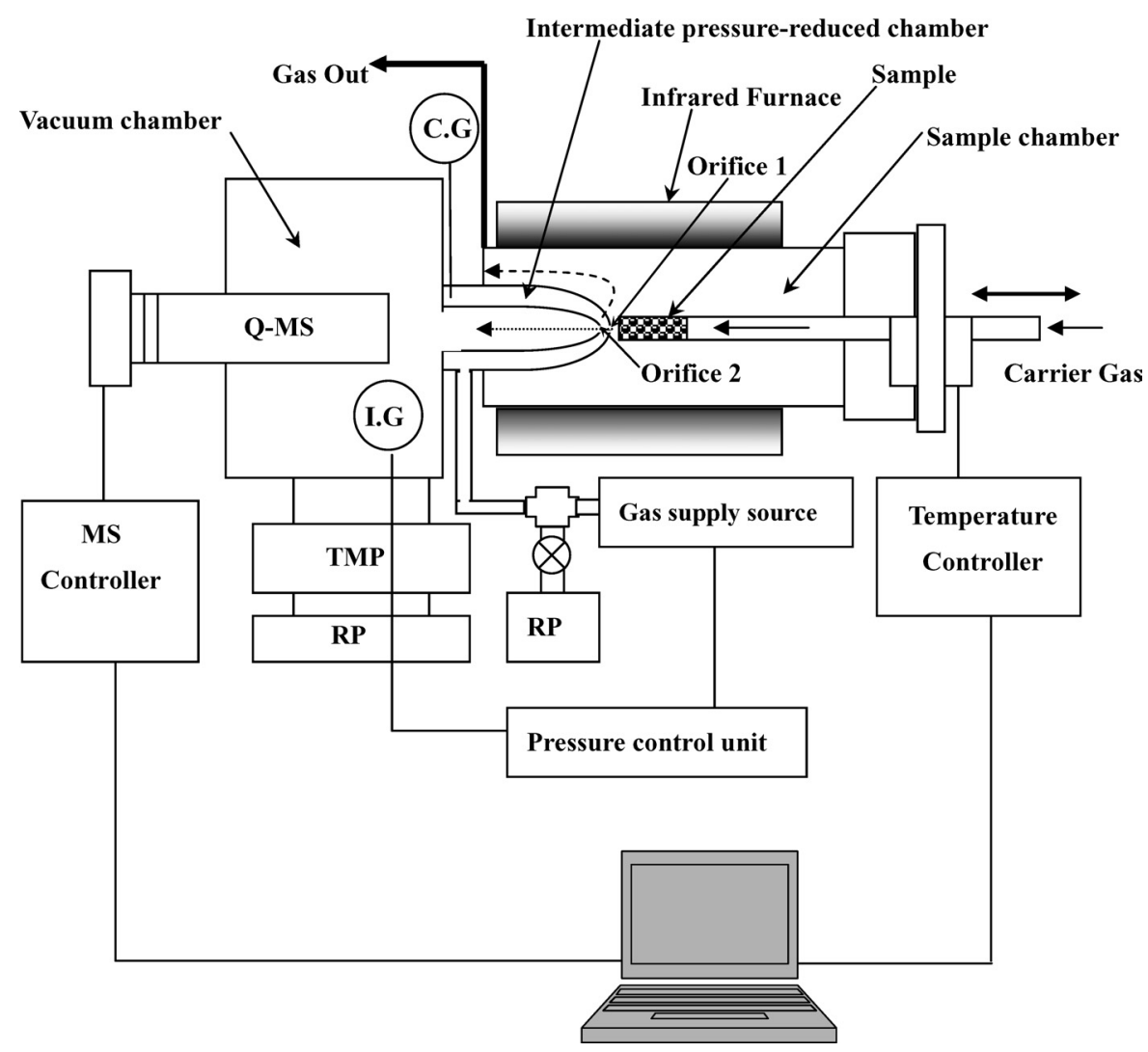

Fig. 1. Schematic diagram of EGA-MS using skimmer interface system with PCF. Ion gauge, I.G; Crystal gauge, C.G; Turbo molecular pump, TMP; Rotary pump, RP. 
controlled to be fixed by the pressure control unit, whereby the pressure variation by the evolved gas introduced through the first orifice from the sample is carried out by combination of adjusting exhaust speed of a vacuum pump and leak-gas speed from a gas supply source so that the output signal of the ion gauge may be kept at constant value. Therefore, the present pressure control function is characterized in that the pressure in the MS chamber is controlled to be fixed at all times during the thermal analysis. As a result, the pressure in the MS chamber is satisfactorily stabilized, and the reduction in detection sensitivity of the MS for the evolved gas can be suppressed.

2.2.2 TG-MS The spectra of the gaseous products evolved from the specimen in thermogravimetricdifferential thermal analysis (TG-DTA; Model Thermo Plus 8120D, Rigaku) are simultaneously monitored with a quadrupole mass spectrometer (Q-MS; Model MQA-200TS, Anelva). ${ }^{14)}$ The Q-MS system is connected to TG-DTA via a gas transfer tube with fused silica capillary tube of an internal diameter of $0.075 \mathrm{~mm}$. All of the transfer pathway was kept at $280^{\circ} \mathrm{C}$ to minimize condensation of the gaseous products evolved from the specimen. In a series of the simultaneous TG-MS experiments, to ignore desorption of any components adsorbed in the reference material such as $\alpha-\mathrm{Al}_{2} \mathrm{O}_{3}$, only an empty aluminum crucible was used as reference.

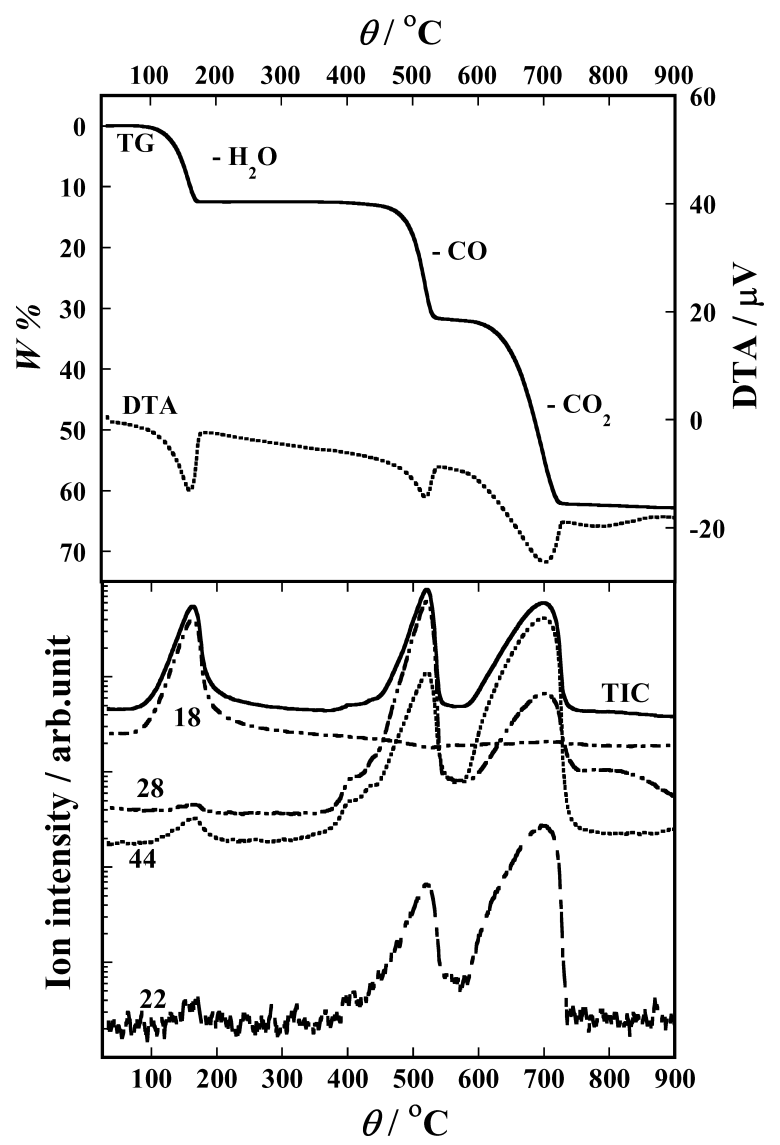

Fig. 2. Typical TG-DTA-MS results of $\mathrm{CaC}_{2} \mathrm{O}_{4} \cdot \mathrm{H}_{2} \mathrm{O}$ in dry helium flow at $10^{\circ} \mathrm{C} / \mathrm{min}$.

\section{Results and Discussions}

\subsection{Improvement of EGA-MS detection sensitivity} by pressure control function

The standard calibration material used in thermogravimetry, calcium oxalate monohydrate $\mathrm{CaC}_{2} \mathrm{O}_{4}$. $\mathrm{H}_{2} \mathrm{O}$, was examined. Figure 2 illustrates the typical TG-MS results of $\mathrm{CaC}_{2} \mathrm{O}_{4} \cdot \mathrm{H}_{2} \mathrm{O}$ : the MS data are represented as the ion chromatograms of $m / z 18,22,28$, and 44 corresponding to $\mathrm{H}_{2} \mathrm{O}^{+}, \mathrm{CO}_{2}^{2+}, \mathrm{CO}^{+}$, and $\mathrm{CO}_{2}^{+}$, respectively. A logarithmic plot allows three decades of intensity to be observed but the shape of the peaks can be directly compared with the derivative TG (DTG) curve when both are plotted on a linear scale. $\mathrm{CaC}_{2} \mathrm{O}_{4}$. $\mathrm{H}_{2} \mathrm{O}$ decomposes in three well-resolved stages ranging from $100^{\circ} \mathrm{C}$ up to $800^{\circ} \mathrm{C}$ as follows.

$$
\begin{aligned}
& \mathrm{CaC}_{2} \mathrm{O}_{4} \cdot \mathrm{H}_{2} \mathrm{O} \rightarrow \mathrm{CaC}_{2} \mathrm{O}_{4}+\mathrm{H}_{2} \mathrm{O} \uparrow \\
& \mathrm{CaC}_{2} \mathrm{O}_{4} \rightarrow \mathrm{CaCO}_{3}+\mathrm{CO} \uparrow
\end{aligned}
$$$$
\mathrm{CaCO}_{3} \rightarrow \mathrm{CaO}+\mathrm{CO}_{2} \uparrow
$$

As an evaluation on the efficiency and the ability of the EGA-MS system, $\mathrm{CaC}_{2} \mathrm{O}_{4} \cdot \mathrm{H}_{2} \mathrm{O}$ was applied under the same experimental conditions. Figure 3 shows a comparison of TIC curves together with the pressure values obtained by the EGA-MS with PCF and without PCF. Although temperatures at peaks of both TIC curves between with PCF and without PCF were in excellent agreement and those temperatures were agreed with temperatures at the inflection points of the TG-MS curve in Fig. 2, a difference of TIC intensities between with PCF and without PCF was clearly observed. Because of the results of the EGA-MS without PCF as shown by dotted line in Fig. 3, actually, we can see that the pressure in the MS chamber was markedly decreased with raising the temperature. As the result, the amount of the evolved gas introduced into the MS through the orifices from the sample was decreased with increasing the temperature. The pressure variation was observed up to $900^{\circ} \mathrm{C}$ in the range of $3.5 \times$ $10^{-3}-1.0 \times 10^{-4} \mathrm{~Pa}$. By adapting of the pressure control function for the EGA-MS, on the other hand, we can see that since the pressure variation in the MS chamber can be stabilized at approximately $3.5 \times 10^{-3} \mathrm{~Pa}$ during the thermal analysis as shown by solid line in Fig. 3,

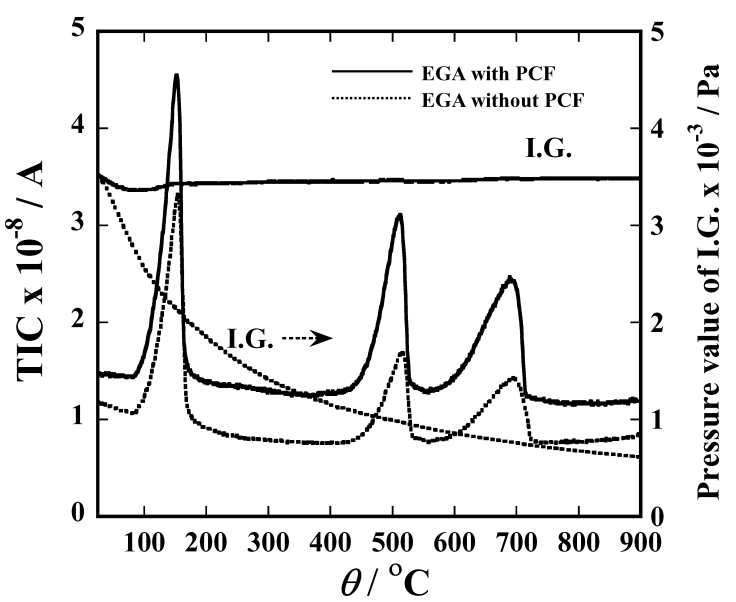

Fig. 3. Comparison of TIC curves together with pressure values obtained by EGA-MS with PCF and without PCF. 
Table 1. Comparison of Peak Areas of Ion Intensities Obtained by EGA-MS with PCF and without PCF of $\mathrm{CaC}_{2} \mathrm{O}_{4} \cdot \mathrm{H}_{2} \mathrm{O}$

\begin{tabular}{clcccc}
\hline \multirow{2}{*}{ Step } & \multirow{2}{*}{ Reaction } & & \multicolumn{3}{c}{ Peak area $\times 10^{-4} / \mathrm{A} \cdot \mathrm{s} / \mathrm{g}$} \\
\cline { 4 - 6 } & & & With $\mathrm{PCF}^{\text {a) }}\left(\mathrm{A}_{1}\right)$ & Without $\mathrm{PCF}^{\text {a) }}\left(\mathrm{A}_{2}\right)$ & Ratio $\left(\mathrm{A}_{1} / \mathrm{A}_{2}\right)$ \\
\hline 1 & $\mathrm{CaC}_{2} \mathrm{O}_{4} \cdot \mathrm{H}_{2} \mathrm{O} \rightarrow \mathrm{CaC}_{2} \mathrm{O}_{4}+\mathrm{H}_{2} \mathrm{O} \uparrow$ & 18 & 4.386 & 3.518 & 1.518 \\
2 & $\mathrm{CaC}_{2} \mathrm{O}_{4} \rightarrow \mathrm{CaCO}_{3}+\mathrm{CO} \uparrow$ & 28 & 3.812 & 1.561 & 2.442 \\
3 & $\mathrm{CaCO}_{3} \rightarrow \mathrm{CaO}+\mathrm{CO}_{2} \uparrow$ & 44 & 4.339 & 2.031 & 2.136 \\
\hline
\end{tabular}

a) Pressure Control Function $(\mathrm{PCF})$

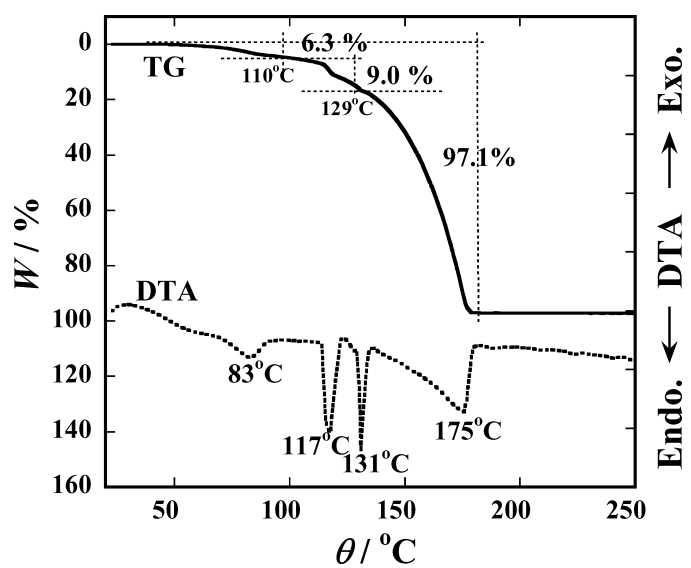

Fig. 4. Typical TG-DTA results of $\mathrm{C}_{10} \mathrm{H}_{14} \mathrm{O}_{4} \mathrm{Zn} \cdot \mathrm{H}_{2} \mathrm{O}$ in dry helium flow at $10^{\circ} \mathrm{C} / \mathrm{min}$.

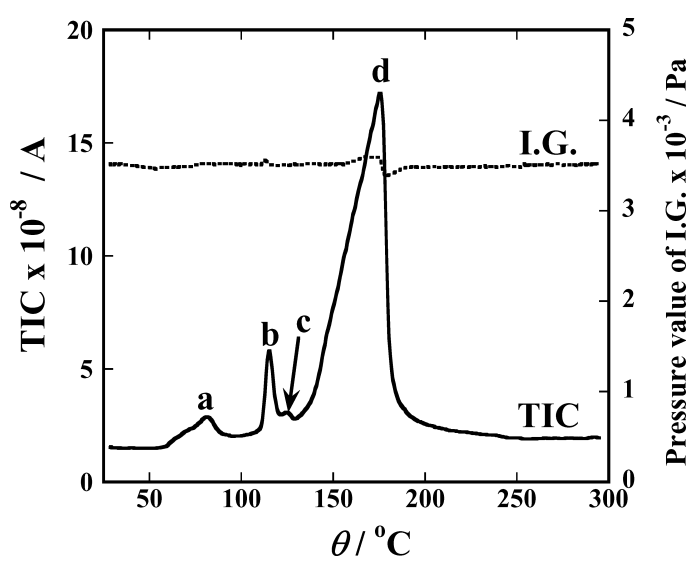

Fig. 5. Typical EGA-MS results of $\mathrm{C}_{10} \mathrm{H}_{14} \mathrm{O}_{4} \mathrm{Zn} \cdot \mathrm{H}_{2} \mathrm{O}$ in dry helium flow at $10^{\circ} \mathrm{C} / \mathrm{min}$.

the reduction in detection sensitivity of the MS to the evolved gas can be suppressed. The ratios of the peak areas corresponding to $\mathrm{m} / z 18,28$, and 44 extracted from both TIC data are shown in Table 1 . As the results, it was proved that the pressure control function contributed remarkably to detect more than 2 times amount of $\mathrm{CO}$ and $\mathrm{CO}_{2}$ evolved above $400^{\circ} \mathrm{C}$.

\subsection{Applications}

3.2.1 Thermal process of $\mathrm{C}_{10} \mathrm{H}_{14} \mathrm{O}_{4} \mathrm{Zn} \cdot \mathrm{H}_{2} \mathrm{O}$ in inert atmosphere Zinc acetylacetonate monohydrate $\left(\mathrm{C}_{10} \mathrm{H}_{14} \mathrm{O}_{4} \mathrm{Zn} \cdot \mathrm{H}_{2} \mathrm{O}\right)$ has attracted much attention as one of the source materials for $\mathrm{ZnO}$ that formed by using metal organic chemical vapor deposition (MOCVD) ${ }^{5)}$ and spray pyrolysis. ${ }^{16)}$ Therefore, it is interesting to study this zinc acetylacetonate precursor and its decomposition process to form $\mathrm{ZnO}$.

The typical TG-DTA curves for $\mathrm{C}_{10} \mathrm{H}_{14} \mathrm{O}_{4} \mathrm{Zn} \cdot \mathrm{H}_{2} \mathrm{O}$ at a heating rate of $10^{\circ} \mathrm{C} / \mathrm{min}$ in dry helium flow are shown in Fig. 4. The thermal process was exhibited via sever-
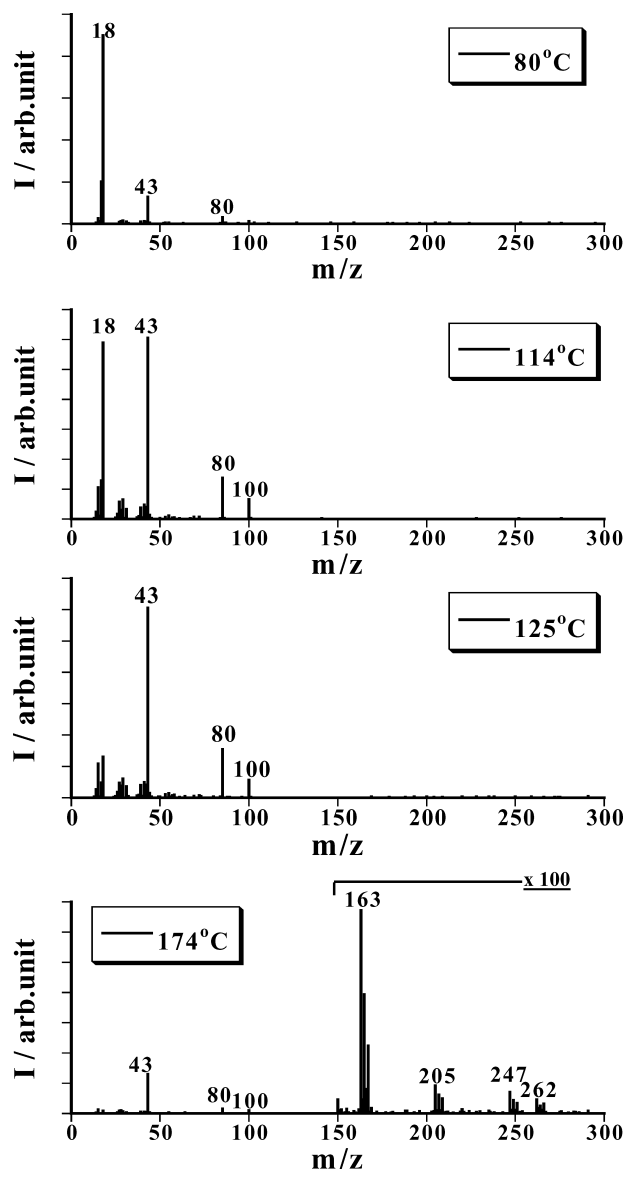

Fig. 6. Comparison of mass spectra obtained at $80^{\circ} \mathrm{C}$, $114^{\circ} \mathrm{C}, 125^{\circ} \mathrm{C}$, and $174^{\circ} \mathrm{C}$ corresponding to peaks of a, b, c, and d, respectively, in Fig. 5 in dry helium atmosphere.

al complex steps; a small amount of residue in a sample pan was observed after the thermal analysis. The first mass loss of $6.3 \%$ up to $110^{\circ} \mathrm{C}$ accompanying a broad endothermic DTA peak at $82^{\circ} \mathrm{C}$ was attributed to dehydration of one water molecule (6.40\%). After dehydration, the mass loss was divided into three reaction steps by the inflection points resolved from the DTG curve. The total mass loss reaches over $97.1 \%$ at its final point $\left(200^{\circ} \mathrm{C}\right)$ and presumably the small amount of $\mathrm{ZnO}$ was formed as residue. On the other hand, the DTA curve indicated four endothermic peaks of $83^{\circ} \mathrm{C}$, $117^{\circ} \mathrm{C}, 131^{\circ} \mathrm{C}$, and $175^{\circ} \mathrm{C}$, respectively. The first endothermic DTA peak accompanying the mass loss was broad, whereas the second and the third endothermic DTA peaks were very sharp, followed by the relatively broad peak appeared on DTA curve. The shape of the second and the third DTA peaks seem to be indicated that phase transition or melting phenomena of anhy- 
drous zinc acetylacetonate occurred. The final large mass loss accompanying the fourth endothermic peak at $175^{\circ} \mathrm{C}$ was probably attributed to evaporation or sublimation of the specimen. Such EGA-MS analysis of the specimen including high boiling point compounds in the evolved gas stream has a significant problem, because the gas sampling interface system has generally a problem of adsorption of the evolved gaseous molecules.

The typical EGA-MS curves for $\mathrm{C}_{10} \mathrm{H}_{14} \mathrm{O}_{4} \mathrm{Zn} \cdot \mathrm{H}_{2} \mathrm{O}$ at a heating rate of $10^{\circ} \mathrm{C} / \mathrm{min}$ in dry helium flow are shown in Fig. 5, where the TIC curve indicates sum of the ion current ranging from $\mathrm{m} / z 10$ to 300 for all detected species. Figure 6 shows a comparison of the mass spectra obtained at the TIC peaks $(a-d)$ during the mass losses, where the mass spectra indicate the signal intensities subtracting the background spectrum obtained before heating. The observed mass spectra obtained at the first to third TIC peaks seem to be similar to each other, suggesting the same components were evolved concurrently. Intense ion peaks at $\mathrm{m} / z 17$ and 18 observed in the mass spectrum corresponding to the first TIC peak at $80^{\circ} \mathrm{C}$ (a) revealed that water vapor was mainly evolved by dehydration. The mass spectra corresponding to the second and third peaks of TIC curve at $114^{\circ} \mathrm{C}$ (b) and $125^{\circ} \mathrm{C}$ (c), respectively, during the second mass loss of $9.0 \%$ consisted of a set of ion peaks at $43,58,72,85$, and 100 . By comparing this set of ion peaks with NIST database, the original source could be identified as acetylacetone $(\mathrm{m} / z 15,29,43,58$, 72,85 , and 100), ${ }^{17)}$ suggesting the decomposition of the specimen. The other weak ion peaks corresponded approximately to the fragmentation ions of acetylacetone, and water vapor. The mass spectrum in the final mass loss stage corresponding to the fourth TIC peak at around $174^{\circ} \mathrm{C}$ (d) was quite different from those in other stages and indicated the set of ion peaks in the higher $m / z$ range, such as $m / z 163,205,247$, and 262 . The simultaneous detection of ions of $m / z 43,163,205$, 247 , and 262 indicated evolution of anhydrous zinc acetylacetonate. ${ }^{18)}$ This fact suggested sublimation or evaporation of the specimen. However, we can learn that the thermal decomposition of the specimen took place concurrently because the intensity of $\mathrm{m} / z 43$ and 163 ions was fairly higher than expected intensities of the fragmentation ions of anhydrous zinc acetylacetonate.

Figure 7 exhibits the identified ion intensities of $\mathrm{m} / \mathrm{z}$ $18\left(\mathrm{H}_{2} \mathrm{O}^{+}\right), 43\left(\mathrm{CH}_{3} \mathrm{CO}^{+}\right), \quad 100\left(\mathrm{C}_{5} \mathrm{H}_{8} \mathrm{O}_{2}^{+}\right)$, and 262 $\left(\mathrm{C}_{10} \mathrm{H}_{14} \mathrm{O}_{4} \mathrm{Zn}^{+}\right)$as a function of the temperature. It was proposed that sublimation and decomposition of anhydrous zinc acetylacetonate began simultaneously after the third TIC peak (c) was observed at temperature over $130^{\circ} \mathrm{C}$.

3.2.2 Thermal degradation of polymethylmethacrylate (PMMA) in inert atmosphere Pyrolysis mass spectrometry has been utilized as a simple and convenient means for studying the composition of polymeric systems with minimal sample preparation. Inspection of the mass spectra of pyrolysis products or pyrolyzates usually gives positive identification of the precursors in polymeric system. Insource pyrolysis does not involve intermediate transfer steps between pyrolyzer

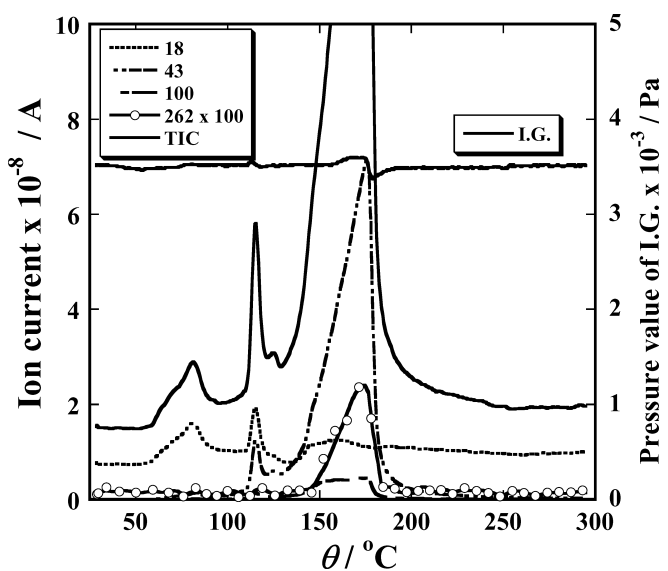

Fig. 7. Mass chromatograms of characteristic ions of $\mathrm{m} / \mathrm{z} 18,43,100$, and 262 obtained in dry helium atmosphere.

$m / z \quad 18, \quad \mathrm{H}_{2} \mathrm{O}^{+} ; \quad m / z \quad 43, \mathrm{CH}_{3} \mathrm{CO}^{+}, \quad m / z \quad 100$ $\mathrm{C}_{5} \mathrm{H}_{8} \mathrm{O}_{2}^{+} ; m / z 262, \mathrm{C}_{10} \mathrm{H}_{14} \mathrm{O}_{4} \mathrm{Zn}^{+}$.
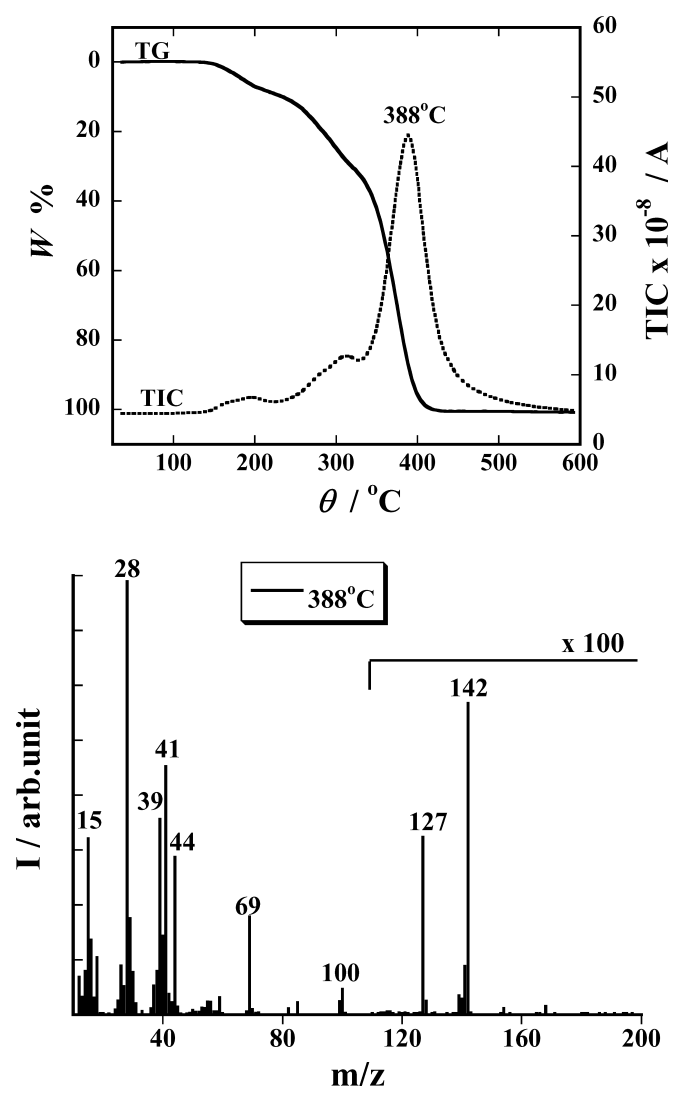

Fig. 8. Typical TG-MS results at $20^{\circ} \mathrm{C} / \mathrm{min}$ for PMMA in helium atmosphere and mass spectrum obtained at TIC peak temperature $\left(388^{\circ} \mathrm{C}\right)$.

and MS and allows the analysis of all constituents of a polymeric system, provided they can be volatilized. Differences in volatility among the constituents can be exploited to heat the sample to pyrolytic temperatures. However, possibility that the activated pyrolyzates passing through the interface system may be changed has been sometimes pointed out by the results of MS.

When degraded alone in the pure state, PMMA breaks down in a very simple manner. It has been established that homolysis occurs at some points in the polymer backbone and this is followed by depolymeri- 

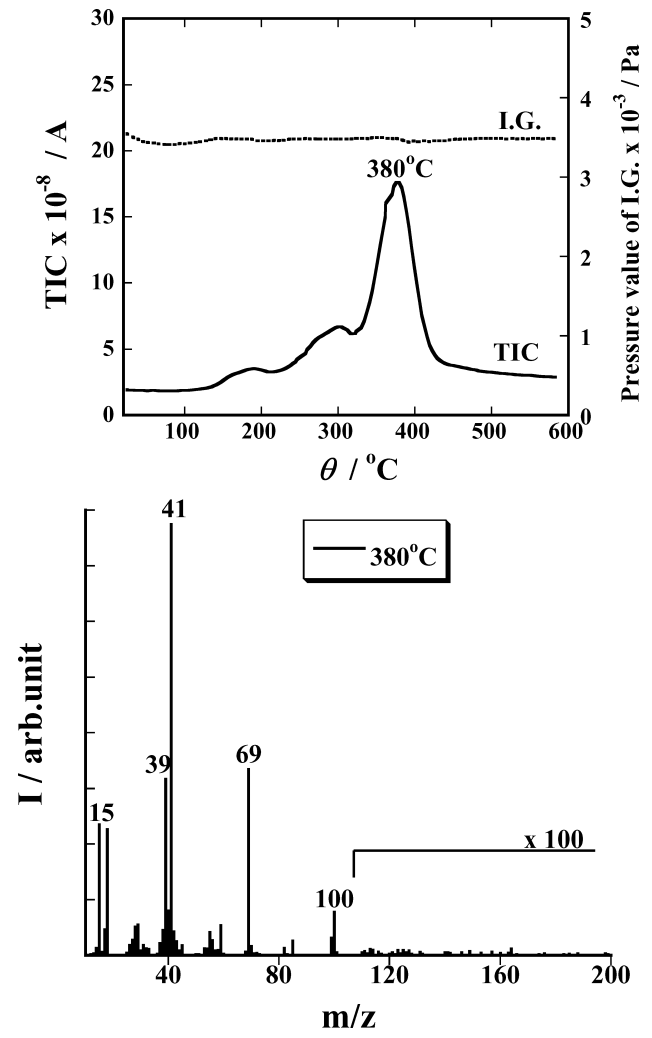

Fig. 9. Typical EGA-MS results at $20^{\circ} \mathrm{C} / \mathrm{min}$ for PMMA in helium atmosphere and mass spectrum obtained at TIC peak temperature $\left(380^{\circ} \mathrm{C}\right)$.

zation to monomer. ${ }^{19)}$

Figure 8 illustrates the typical TG-MS results for PMMA at a heating rate of $20^{\circ} \mathrm{C} / \mathrm{min}$ in dry helium flow and the mass spectrum obtained at the main TIC peak $\left(388^{\circ} \mathrm{C}\right)$. The mass loss was characterized through three degradation stages in which the mass spectra obtained at the TIC peaks were identified as methylmethacrylate (MMA) $(m / z 15,39,41,69$, and 100) by agreement with NIST database except for $m / z 28,44$, 127 , and 142. These results indicate that the observed ions of $m / z 28,44,127$, and 142 may be interpreted as ions of those of the by-products formed in the gas sampling interface system, if the degradation is only controlled by depolymerization to monomer. In other words, the part of the evolved gas could be denatured in the interface system which must be maintained at high temperature to avoid condensation of the activated MMA pyrolyzates colliding with the inner wall of the long capillary tube.

In order to solve the above-mentioned speculation, the degradation of PMMA was inspected by the EGA-MS equipped with the skimmer interface system which does not principally caused such sub-reactions in the interface system. Figure 9 illustrates typical EGA-MS curves for PMMA at the peak of the TIC curve at a heating rate of $20^{\circ} \mathrm{C} / \mathrm{min}$ in dry helium flow and the mass spectrum obtained at the peak of the TIC curve $\left(380^{\circ} \mathrm{C}\right)$. The thermal behavior agreed satisfacto- rily with that of the TG-MS result shown in Fig. 8: the three degradation steps were observed. The mass spectrum was perfectly in agreement with that of MMA in NIST-MS database and additional ions originated in the by-products were not found in Fig. 8. Finally, we can learn that the activated MMA pyrolyzates degraded from PMMA were directly introduced without any change into the MS through the skimmer interface system.

\section{Conclusions}

It has been confirmed that new EGA-MS equipped with both the skimmer interface system and the pressure control function are effective to enhance the detection sensitivity in a higher mass range and a wide temperature range. The EGA-MS was evaluated by the thermal decomposition of calcium oxalate monohydrate, zinc acetylacetonate monohydrate and polymethylmethacrylate. It is concluded that the more sensitive and precise gas analysis which prevented condensation of evolution gases and/or generation of by-products can be performed, by comparing with the capillary interface system of TG-MS.

\section{References}

1) J. Chiu and A. J. Beattie, Thermochim. Acta, 50, 49 (1981).

2) M. Muller-Vonmoos, G. Kahr, and A. Rub, Thermochim. Acta, 20, 387 (1977).

3) B. B. Johanson and J. Chiu, Thermochim. Acta, 50, 57 (1981).

4) M. Mittleman, Thermochim. Acta, 166, 301 (1990).

5) J. J. Morelli, J. Anal. Appl. Pyrolysis, 18, 1 (1990).

6) F. Isa, H. Hasegawa, and T. Arii, Netsu Sokutei, 22, 160 (1995).

7) T. Tsugoshi, M. Furukawa, M. Ohashi, and Y. Iida, J. Therm. Anal. Cal., 64, 1127 (2001).

8) A. R. McGhile, Thermochim. Acta, 234, 21 (1994).

9) T. Arii, T. Senda, and N. Fujii, Thermochim. Acta, 267, 209 (1995).

10) T. Arii, J. Mass Spectrom. Soc. Jpn., 46, 374 (1998).

11) E. Kaisersberger and M. G. Gebhardt, Thermochim. Acta, 148, 493 (1989).

12) Entry No. 20-0231, ICDD (calcium oxalate monohydrate, $\mathrm{CaC}_{2} \mathrm{O}_{4} \cdot \mathrm{H}_{2} \mathrm{O}$ ).

13) Entry No. 23-1970, ICDD (zinc acetylacetonate, $\mathrm{C}_{10} \mathrm{H}_{14} \mathrm{O}_{4} \mathrm{Zn}$ ).

14) T. Arii, K. Terayama, and N. Fujii, J. Therm. Anal., 47, 1649 (1996).

15) T. Minami, H. Sonohara, S. Taketa, and H, Sato, Jpn. J. Appl. Phys., 33, L743 (1994).

16) A. J. C. Fiddes, K. Durose, A. W. Brinkman, J. Woods, P. D. Coates, and A. J. Banister, J. Crystal Growth, 159, 1996 (1996).

17) Entry No. 19843, NIST 107 (acetylacetone, $\mathrm{C}_{5} \mathrm{H}_{8} \mathrm{O}_{2}$ ).

18) Entry No. 12767, NIST 107 (zinc acetylacetonate, $\left.\mathrm{C}_{10} \mathrm{H}_{14} \mathrm{O}_{4} \mathrm{Zn}\right)$.

19) I. C. McNell, Eur. Polym. J., 4, 21 (1968).

Keywords: EGA-MS, Skimmer interface, Pressure control function, Capillary interface, Thermal decomposition 